\title{
Modelling frequency-dependent anisotropy due to fluid flow in bed limited cracks
}

Simon R. Tod*, John A. Hudson, Department of Applied Mathematics and Theoretical Physics, University of Cambridge and Enru Liu, Edinburgh Anisotropy Project, British Geological Survey

\section{Summary}

Evidence from a number of measurements support the idea that anisotropy, or shear-wave splitting, exhibits a frequency dependence, that is generally attributed to properties of the microstructure of the rock. This effect is generally assumed to be the result of scattering from oriented inclusions within the rock mass, however there are a number of competing mechanisms that may give rise to this observed frequency dependence. The scale length of the inclusions must be much smaller than the wavelength at which the measurements where conducted, in order for their presence to be observed as an effective anisotropy, and may therefore be insufficient to account for a significant frequency dependence. An alternative mechanism resulting in frequency dependence is the transfer of fluid between the inclusions, assumed to be fluid filled. Using an established model, it is demonstrated that this fluid effect is potentially significant enough to explain observed frequency dependence.

\section{Introduction}

There is evidence from measurements taken from a range of experiments that shear wave splitting exhibits a frequency dependence (e. g. Marson-Pidgeon and Savage, 1997; Lynn et al., 1999). Marson-Pidgeon and Savage (1997) and Matcham et al. (2000), see Fig. 1, indicate that oriented inclusions are the most likely cause of the observed results in the earthquake data, and the seismic data of Lynn et al. (1999) and Liu et al. (2002), see Fig. 2, is modelled by Chesnokov et al. (2001) in terms of scattering from such. To achieve results in the modelling comparable to those from the data it was found necessary to use a high concentration of large inclusions. These values are at levels close to, or beyond, the range of validity of effective medium theories.

Effective medium theories have been developed that give the overall properties of an elastic material containing cracks by a number of authors (e. g. O,Connell and Budiansky, 1974; Hudson, 1980). These typically require the description of a mean crack shape. In general, for simplicity, this is taken to be a flat circular ("penny-shaped") crack, though it may also be elliptical (Budiansky and O'Connell, 1976). However, these restrictions place an unnecessary limitation on the theory, when it is perhaps more realistic to describe a crack in terms of a bounded width and an otherwise ellipsoidal shape. Such ellipsoids of non-zero thickness have been shown to give rise to more general formulations (e. g. Kuster and Toksöz, 1974; Hudson, 1994) and nonelliptical thin cracks have also been studied (e. g. Mavko and Nur, 1978).

A generalization of the method of smoothing (Hudson, 1994) has been used to study the role of the crack width and the ratio of the two larger dimensions in determining the properties of

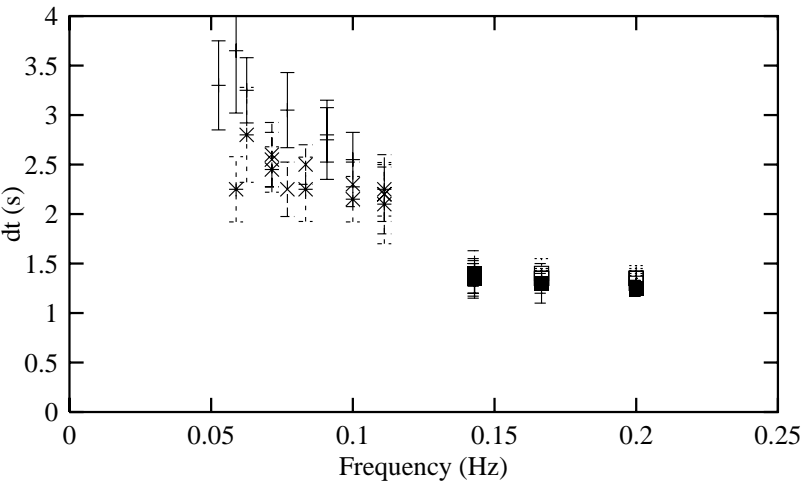

Fig. 1: Delay time as a function of frequency for three SKS and two ScS events recorded at a broadband station in Wellington, New Zealand. Data reproduced with permission from K. Marson-Pidgeon and M. K Savage, from Fig. 4 of Marson-Pidgeon and Savage (1997), originally displayed as a function of frequency.

the effective medium. This results in an orthorhombic medium, and provides a suitable description of a material where the crack dimensions are restricted in one direction due to, for example, bed-limiting effects, while remaining unconfined in other directions. In addition to the isolated crack description, the theory is extended to include the fluid flow mechanism of Tod (2001) that models the flow as being dominated by crack to crack flow and is valid for low matrix porosities over a large range of frequencies, provided that the wavelength is much greater than the crack dimensions. The resulting theory predicts a frequency dependence in the elastic stiffnesses as a result of the effects of fluid flow.

\section{Data interpretation}

The broadband earthquake data from New Zealand, shown in Fig. 1, covers a range of frequencies below $0.1 \mathrm{~Hz}$. The original figure given by Marson-Pidgeon and Savage (1997) was of time delay against period, while we have replotted the data against frequency. While the large errors bars at low frequency leave the interpretation a little fragile there is a clear decrease in delay time with frequency, of a roughly linear nature, with perhaps the suggestion of a peak, non-zero, frequency before a slight decrease for the lowest frequencies.

The VSP from the Bluebell-Altamont Field, Utah, shows a limited coverage of the seismic frequency range and the three data points given by Chesnokov et al. (2001) suggest a monotonic decrease in shear wave splitting with frequency, so that maximum anisotropy is attained in the static limit, in agreement with the scattering theory. A more comprehensive analysis of the same data by Liu et al. (2002), shown in Fig. 2, suggests that this trend is perhaps not quite so simple. While one may question, due to the limited frequency coverage of the data, the two points 


\section{Frequency-dependent anisotropy}

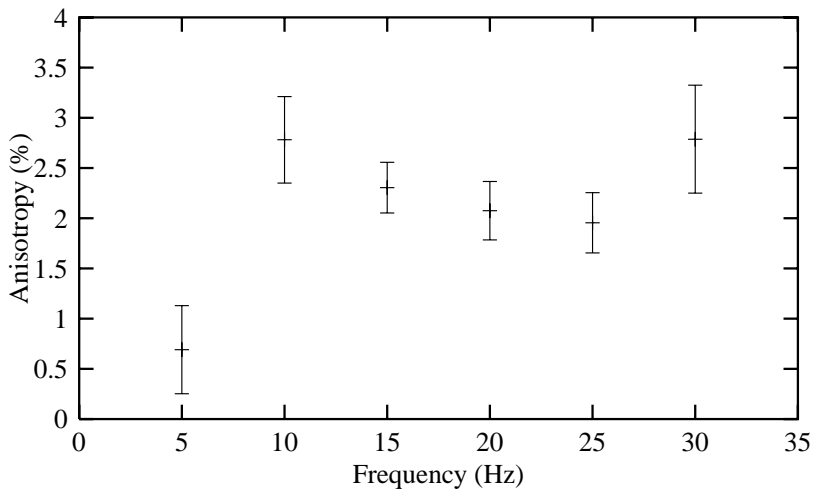

Fig. 2: The variation in shear wave anisotropy with frequency from a VSP in the Bluebell-Altamont Field, Utah. Data reproduced with permission from H. Lynn, Lynn Inc.

that buck the aforementioned trend, there is a possible suggestion that the maximum anisotropy is achieved at a low, non-zero, frequency and that the behaviour at high frequencies is not quite as simple as it might at first seem.

\section{Theory}

The effective medium theory due to Hudson (1980) and based on the method of smoothing (Keller, 1964), was extended by Hudson (1994) to include cracks of finite width, modelled as ellipsoids with semi-axis $a, b$ and $c$. The elastic stiffnesses $\mathbf{c}$ of the resulting effective medium for a single set of aligned cracks of identical dimensions are of the form

$$
\mathbf{c}=\mathbf{c}^{0}+s \mathbf{c}^{+} \mathbf{E},
$$

where $\mathbf{c}^{0}$ are the elastic stiffnesses of the isotropic matrix material, $s=4 \pi \nu^{s} a b c / 3$ is the volume fraction of cracks, which we may identify as the porosity of the cracks, in terms of the number density $\nu^{s}$,

$$
\mathbf{c}^{+}=\mathbf{c}^{\prime}-\mathbf{c}^{0}
$$

in terms of the elastic stiffnesses of the inclusions $\mathbf{c}^{\prime}$, and $\mathbf{E}$ is defined in terms of the Eshelby (1957) tensor $\mathbf{S}$ as

$$
\mathbf{E}=\left(\mathbf{S s}^{0} \mathbf{c}^{+}+\mathbf{I}\right)^{-1}
$$

where $\mathbf{s}^{0}$ are the elastic compliances of the matrix material and $\mathbf{I}$ is the identity tensor. c corresponds to an orthorhombic medium. For multiple crack sets, the perturbation to the background stiffnesses $\mathbf{c}^{0}$ can be found by summing over contributions from each of the sets. $\mathbf{E}$ relates the strain in an inclusion $\mathbf{e}$ to the imposed strain at infinity $\mathbf{e}^{0}$,

$$
\mathbf{e}=\mathbf{E e}^{0} .
$$

The effective density of the medium is

$$
\rho=\rho^{0}(1-s)+\rho^{\prime} s,
$$

where $\rho^{0}$ and $\rho^{\prime}$ are the density of the matrix and inclusion respectively.
To determine the strain in an inclusion due to an imposed stress at infinity $\boldsymbol{\sigma}^{0}$ and a fluid pressure $p_{f}$ within the inclusion we write, following Tod (2002a),

$$
\mathbf{e}=\hat{\mathbf{E}} \mathbf{e}^{0}
$$

where

$$
\hat{E}_{i j k l}=\bar{E}_{i j k l}+\left(\bar{E}_{i j r s} s_{r s p p}^{0}-\frac{\delta_{i j}}{3 \kappa}\right) H_{m n} c_{m n k l}^{0},
$$

with

$$
\overline{\mathbf{E}}=(\mathbf{I}-\mathbf{S})^{-1}
$$

the result for dry cracks, $\kappa$ the bulk modulus of the matrix material and $\mathbf{H}$ arising from a linearized relation of the form

$$
p_{f}=H_{i j} \sigma_{i j}^{0}
$$

derived from the fluid flow model of Hudson et al. (1996) and Tod (2001), and given explicitly by Tod (2002a), valid for low matrix porosities.

On the assumption that the cracks are all fully aligned and are of identical dimension we may write (Tod, 2002a)

$$
\hat{E}_{i j k l}=\bar{E}_{i j k l}+\frac{\kappa_{f}}{3 \kappa}\left(\bar{E}_{i j u u}-\delta_{i j}\right) \chi \bar{E}_{v v k l},
$$

where

$$
\begin{gathered}
\chi=\frac{1}{1-\mathrm{i} \omega \tau \gamma}\left[\mathrm{i} \omega \tau-\frac{1}{\gamma+\mathrm{i} \omega \tau K_{2}(1-\mathrm{i} \omega \tau \gamma)}\right] \\
\gamma=1-\frac{\kappa_{f}}{\kappa}+\frac{\kappa_{f}}{3 \kappa} \bar{E}_{i i j j}
\end{gathered}
$$

and

$$
K_{2}=\frac{\kappa_{f} K^{r}}{s v^{2} \tau \eta_{f}}
$$

for an isotropic in situ rock permeability $K^{r}$, after Tod (2002b) $\kappa_{f}$ and $\eta_{f}$ are the bulk modulus and viscosity of the fluid, $v$ is taken to be a lowest order approximation to the wavespeed, and $\tau$ is a relaxation parameter that characterises the time-scale of pressure equalization between neighbouring cracks. Hudson et al. (1996) estimate this to be

$$
\tau=\frac{\phi^{m} \eta_{f} l^{2}}{\kappa_{f} K^{m}}
$$

in terms of a characteristic length scale $l$, the matrix permeability $K^{m}$ and the matrix porosity $\phi^{m}$. For the modelling that follows, we replace $\mathbf{E}$ in Eq. (1) with $\hat{\mathbf{E}}$ (Eq. 10).

\section{Modelling}

We start by attempting to reproduce the behaviour shown in the VSP data in Fig. 2. We model the properties of the matrix material as $v_{P}=4.88 \times 10^{3} \mathrm{~m} \mathrm{~s}^{-1}, v_{S}=2.68 \times 10^{3} \mathrm{~m} \mathrm{~s}^{-1}$ and $\rho=2.55 \times 10^{3} \mathrm{~kg} \mathrm{~m}^{-3}$ and take the inclusions to be filled with gas with $v_{P}=0.64 \times 10^{3} \mathrm{~m} \mathrm{~s}^{-1}$ and $\rho=0.18 \times 10^{3} \mathrm{~kg} \mathrm{~m}^{-3}$, as Chesnokov et al. (2001). Furthermore, we know from Lynn 
Frequency-dependent anisotropy

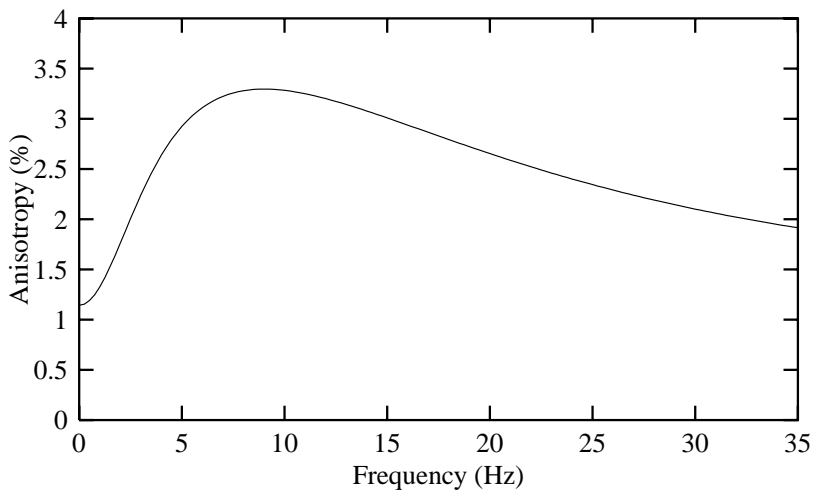

Fig. 3: Modelling the change in shear wave anisotropy with frequency from the VSP in the Bluebell-Altamont Field, Utah. See text for material parameters.

et al. (1999) that $K^{m}<1.0 \times 10^{-15} \mathrm{~m}^{2}$ and $\phi^{m}<0.08$, and we take $\eta_{f}=1.8 \times 10^{-5} \mathrm{~kg} \mathrm{~m}^{-1} \mathrm{~s}^{-1}$ for the gas. We estimate $K^{r}=3.1 \times 10^{-11} \mathrm{~m}^{2}$ and $\tau=1.0 \times 10^{-3}$ s. The parameters still available to us are the volume fraction of cracks, two aspect ratios that describe the shape of the crack and up to three Eulerian angles describing the orientation of the cracks. We use the following values: $s=5.6 \times 10^{-5}, \alpha \equiv c / a=1.0 \times 10^{-5}$, $\beta \equiv b / a=2.5 \times 10^{-2}$ and position the crack normal in the $(1,3)$-plane at $60^{\circ}$ to the vertical. If we describe the (shear wave) anisotropy by

$$
\gamma_{T}=\frac{v_{S}^{\text {fast }}-v_{S}^{\text {slow }}}{v_{S}^{\text {fast }}}
$$

then the change in anisotropy with frequency due to the vertical incidence is given in Fig. 3. This shows the same qualitative behaviour as Fig. 2, with a decrease in anisotropy with frequency from a peak value at around $8 \mathrm{~Hz}$. The data point at $30 \mathrm{~Hz}$, with by far the largest error bar, cannot be explained by the model, though it goes against intuition.

Rather less is know about the in situ measurements appropriate to the earthquake data reproduced in Fig. 1, we may nevertheless aim to achieve a qualitative comparison. Using the same rock properties, but switching to a fluid, such that $v_{P}=1.5 \times 10^{3} \mathrm{~m} \mathrm{~s}^{-1}$ and $\rho=1.0 \times 10^{3} \mathrm{~kg} \mathrm{~m}^{-3}$ then using $s=3.08 \times 10^{-4}, \alpha=1.0 \times 10^{-4}, \beta=3.5 \times 10^{-2}$, $\tau=5.0 \times 10^{-2} \mathrm{~s}$ and $\eta_{f} / K^{r}=1.0 \times 10^{3} \mathrm{~kg} \mathrm{~m}^{-3} \mathrm{~s}^{-1}$, the modelling yields Fig. 4. This shows the same qualitative behaviour as Fig. 1 with the anisotropy drop occurring over the same frequency and being of the same order of magnitude.

While the volume fraction $s$ is present in the non-dimensional parameter $K_{2}$ (Eq. 13) that influences both the frequency at which the peak anisotropy occurs and the magnitude of the peak in comparison with the high frequency limiting value, its major effect is simply to scale the magnitude of the anisotropy. $\tau$ also influences both the location and magnitude of the peak. Changes in the two aspect ratios alter the magnitude of the resulting anisotropy, but has little or no effect on the shape of the variation of the anisotropy with frequency. On the assumption that there is only a single set of aligned cracks present, we may recover the crack orientation from $\mathrm{P}$-wave AVOA analysis of

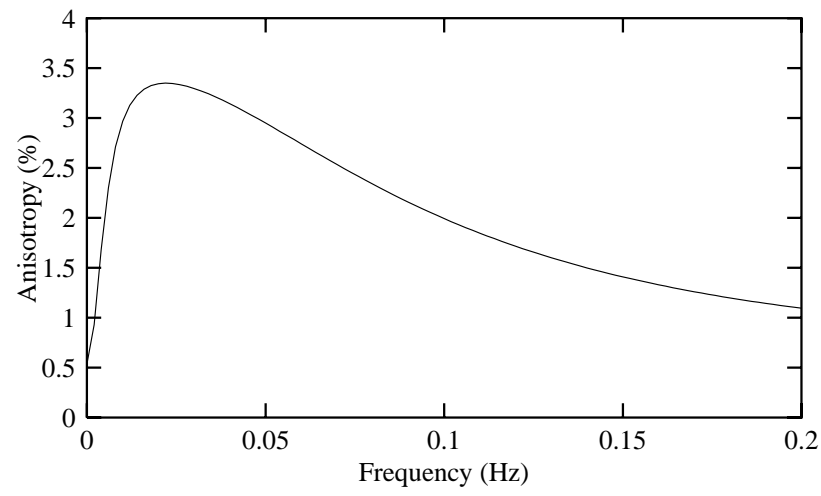

Fig. 4: Modelling the change in shear wave anisotropy with frequency from the broadband data at Wellington, New Zealand. See text for material parameters.

wide azimuth data.

\section{Conclusion}

We have presented a model that describes a fluid flow mechanism between ellipsoidal cracks that produces a frequency dependence in the resulting effective material parameters and hence in the shear wave splitting. The model has been used to simulate field observations of frequency dependence in both exploration and earthquake data sets, with great success. We demonstrate that the effects of fluid flow, whether that be gas or liquid, are non-negligible and an important contributing factor to observed frequency dependent anisotropy. While in general the effects of both fluid flow and scattering need to be taken into account when interpreting the frequency dependence, the effect is likely to be dominated by one process or the other dependent upon scaling arguments.

\section{Acknowledgements}

We would like to thank Katrina Marson-Pidgeon and Martha K. Savage for providing us with the data from Fig. 4 of Marson-Pidgeon and Savage (1997), reproduced in Fig. 1, Heloise Lynn, Lynn Inc., for permission to use data from a 9C VSP in the Bluebell-Altamont Field, Utah, reproduced in Fig. 2, and John H. Queen, Conoco, for useful discussion. The work was sponsored by the Natural Environment Research Council through project GST022305, as part of the thematic programme Understanding the micro-to-macro behaviour of rock fluid systems $(\mu 2 M)$ and is presented with the approval of the Executive Director of the British Geological Survey (NERC).

\section{References}

Budiansky, B., and O'Connell, R. J., 1976, Elastic moduli of a cracked solid: Int. J. Solids Struct., 12, 81-97.

Chesnokov, E. M., Queen, J. H., Vichorev, A. A., Lynn, H. B., Hooper, J. M., Bayuk, I. O., Castagna, J. A., and Roy, B., 


\section{Frequency-dependent anisotropy}

2001, Frequency dependent anisotropy: Frequency dependent anisotropy: 71st Ann. Int. Mtg. SEG, Exp. Abs.

Eshelby, J. D., 1957, The determination of the elastic field of an ellipsoidal inclusion and related problems: Proc. R. Soc. Lond. A, 241, 376-396.

Hudson, J. A., Liu, E., and Crampin, S., 1996, The mechanical properties of materials with interconnected cracks and pores: Geophys. J. Int., 124, 105-112.

Hudson, J. A., 1980, Overall properties of a cracked solid: Math. Proc. Camb. Phil. Soc., 88, 371-384.

1994, Overall properties of materials with inclusions or cavities: Geophys. J. Int., 117, 555-561.

Keller, J. B., 1964, Stochastic equations and wave propagation in random media: Proc. Symp. appl. Math., 16, 145-170.

Kuster, G. T., and Toksöz, M. N., 1974, Velocity and attenuation of seismic waves in two-phase media: Part 1. Theoretical formulations: Geophysics, 39, 587-606.

Liu, E., Queen, J. H., Li, X. Y., Chapman, M., Lynn, H. B., and Chesnokov, E. M., 2002, Analysis of frequency-dependent seismic anisotropy from a multicomponent VSP:, Proc. 10th Int. Work. Siesmic Anisotropy, J. Appl. Geophys., submitted.

Lynn, H. B., Beckham, W. E., Simon, K. M., Bates, C. R., Layman, M., and Jones, M., 1999, P-wave and S-wave azimuthal anisotropy at a naturally fractured gas reservoir, BluebellAltamont Field, Utah: Geophysics, 64, 1312-1328.

Marson-Pidgeon, K., and Savage, M. K., 1997, Frequencydependent anisotropy in Wellington, New Zealand: Geophys. Res. Lett., 24, 3297-3300.

Matcham, I., Savage, M. K., and Gledhill, K. R., 2000, Distribution of seismic anisotropy in the subduction zone beneath the Wellington region, New Zealand: Geophys. J. Int., 140, $1-10$.

Mavko, G. M., and Nur, A., 1978, The effect of nonelliptical cracks on the compressibility of rocks: J. geophys. Res., 83, 4459-4468.

O'Connell, R. J., and Budiansky, B., 1974, Seismic velocities in dry and saturated cracked solids: J. geophys. Res., 79, 54125426.

Tod, S. R., 2001, The effects on seismic waves of interconnected nearly aligned cracks: Geophys. J. Int., 146, 249-263.

2002a, Bed-limited cracks in effective medium theory:, Geophys. J. Int., in review.

$2002 \mathrm{~b}$, The effects of stress and fluid pressure on the anisotropy of interconnected cracks: Geophys. J. Int., 149, 149-156. 